\title{
Effect of solution physical chemistry on the rheological properties of activated sludge
}

\author{
F Dilek Sanin \\ Department of Environmental Engineering, Middle East Technical University, 06531 Ankara, Turkey
}

\begin{abstract}
Knowledge on rheological properties of sludge is important for the design of pumping and transport facilities and as a tool in process control during treatment. Concentrated activated sludge is known to behave as a non-Newtonian material. The contribution to nonNewtonian behaviour is believed to originate from the colloidal properties of solids more than from the molecular properties of the suspension. Considering that the colloidal properties of sludge depend on factors like $\mathrm{pH}$, conductivity, solids concentration and flocculation properties, this study examines the effects of these variables on the rheology of activated sludge. Results show that the rheograms obtained fit best to a power law model indicating that the flow of activated sludge can best be expressed by the pseudoplastic flow model. It is also observed that the apparent viscosity increases sharply with the increase of solids concentration. From the $\mathrm{pH}$ values studied, the one that causes the lowest viscosity is the $\mathrm{pH}$ 5.5. As the $\mathrm{pH}$ increases, the viscosity measured increases considerably. This makes sense considering that the isoelectric point of bacteria is at $\mathrm{pH} 2-4$. Conductivity is also found to affect the viscosity greatly; such that as the conductivity increases, the apparent viscosity decreases. Finally, experimental results reveal that the presence of extracellular polymers and the flocculated nature of activated sludge affect its viscosity.
\end{abstract}

\section{Introduction}

Understanding of the rheological properties of sludge is not only important in the design of pumping and transport facilities but also critical for controlling treatment processes like the polymer dose assessment for sludge conditioning prior to dewatering. A property this important did not receive the deserved attention up until recently, mainly due to the difficulties associated with the measurement of viscosity especially of biological sludges. Activated sludge is a biological suspension of flocs having irregular shapes and various sizes that change over time or when the environmental conditions change. Besides, the concentration of solids present in the slurry is another factor of importance in determining the flow properties. A very thin activated sludge may behave very close to water (Newtonian behaviour) in terms of rheological properties; however, with increasing solids concentration, activated sludge exhibits non-Newtonian flow character. Activated sludge has been identified as either a plastic (Dick and Ewing, 1967; Unno and Akehata, 1985; Dick, 1986) or a pseudoplastic (Behn, 1962; Moeller and Torres, 1997) non-Newtonian fluid.

The contribution to non-Newtonian behaviour is believed to originate from the colloidal properties of solids more than the molecular properties of the suspension (Hiemenz and Rajagopalan, 1997). The colloidal properties of sludge that may affect the rheological characteristics are particle size, shape, flexibility, surface charge, solvation and flocculation. Furthermore, the rheological behaviour of colloidal dispersions is summarised to depend mainly on (Shaw, 1992):

- Viscosity of the dispersion medium

- Particle concentration

- Particle size and shape

- Particle-particle and particle-dispersion medium interactions.

奋+90(312)2102642; fax:+90(312)2101260; e-mail: dsanin@metu.edu.tr Received 6 December 2001; accepted in revised form 21 March 2002.
From these factors, the first one, the dispersion medium viscosity is the simplest to deal with. For activated sludge the dispersion medium is water and being a Newtonian fluid, water has a fixed viscosity at a fixed temperature. The second factor, the solids concentration, has been studied more than the other factors for activated sludge and its effect is pretty well documented (Dick and Ewing, 1967; Dick and Buck, 1985; Lotito et. al., 1997). The third factor, particle size and shape, is a very difficult aspect of activated sludge to study. Activated sludge has a broad particle size distribution, which varies over time and according to the physical and chemical factors like shear and solution chemistry. Particleparticle and particle-dispersion medium interactions also depend on particle properties as well as solution physical chemistry. The last two sets of parameters are pretty complicated and have not been studied adequately.

Considering that the colloidal properties of sludge depend on factors like $\mathrm{pH}$, ionic strength, solids concentration and flocculation properties, this study was undertaken to evaluate the effects of these variables on the rheology of activated sludge.

\section{Materials and methods}

The sludge used in the experiments was a waste activated sludge taken from a municipal wastewater treatment plant. The sludge was concentrated to about $2 \%$ solids by gravity settling. Concentrated sludge was then connected to an aeration pump and aerated during the study; its supernatant was stored in the refrigerator and used to dilute the sludge samples when necessary. The tests were conducted in the course of 3 to $4 \mathrm{~d}$. Sludge samples were discarded at the end of each set of experiments and always a new sample was brought to the laboratory for the next set.

Rheological properties of sludge were determined by using a rotational viscometer (Brookfield LVDVII+ equipped with ultralow viscosity adapter). Viscometer spindle diameter was $2.5 \mathrm{~cm}$, with a gap size of $0.15 \mathrm{~cm}$ between the spindle and the reservoir. Shear stress-shear rate relationships were determined at six different rotational speeds, corresponding to six different shear rates. Shear 
rates studied were $1.8,3.7,7.3,14.7,36.7,73.4 \mathrm{~s}^{-1}$. Both the shear stress-shear rate graphs and the apparent viscosities (defined as the ratio of shear stress to shear rate at a fixed shear rate) were studied. Temperature was kept at $25^{\circ} \mathrm{C}$ during the measurements, and time of measurement was kept constant at $1 \mathrm{~min}$.

To study the effect of solids concentration, each sludge sample was prepared in at least 5 different concentrations by diluting or concentrating the sludge samples in the laboratory. The concentration range studied was between about $2000 \mathrm{mg} \cdot \mathrm{l}^{-1}$ to $18000 \mathrm{mg} \cdot \mathrm{l}^{-1}$.

For studying the effects of $\mathrm{pH}, \mathrm{pH}$ adjustments of the sludge to 5.5, 7.4 and 9.1 were made using either $\mathrm{HCl}$ or $\mathrm{NaOH}$.

Conductivity of activated sludge was adjusted adding the phosphate buffer saline (PBS) salts $\left(\mathrm{NaCl}=4 \mathrm{~g} \cdot \mathrm{l}^{-1} ; \mathrm{KCl}=0.1 \mathrm{~g} \cdot \mathrm{l}^{-1}\right.$; $\left.\mathrm{KH}_{2} \mathrm{PO}_{4}=\left.0.06 \mathrm{~g} \cdot\right|^{-1} ; \mathrm{Na}_{2} \mathrm{HPO}_{4}=\left.0.455 \mathrm{~g} \cdot\right|^{-1}\right)$, into the activated sludge mixed liquor. Three conductivities were studied. The first sample was unadjusted activated sludge. The original conductivity of this activated sludge sample was measured as $970 \mu \mathrm{mho} \cdot \mathrm{cm}^{-1}$. When the PBS salts were added to activated sludge, conductivity became $5620 \mu \mathrm{mho} \cdot \mathrm{cm}^{-1}$. This was used as the second sample. Finally, twice the amount of the salts in PBS solution was added to the same activated sludge and this sample then had a conductivity of $10220 \mu \mathrm{mho} \cdot \mathrm{cm}^{-1}$. Samples with different $\mathrm{pH}$ and conductivities were analysed rheologically at all 6 shear rates and at different solids concentrations since viscosity of sludge is a factor of both the shear rate and the solids concentration.

To investigate the effects of centrifugation and the polymer extraction on rheology, activated sludge samples were subjected to multiple centrifugation runs. Centrifugation was done at $2000 \mathrm{G}$ for $20 \mathrm{~min}$ at $4^{\circ} \mathrm{C}$ in $250 \mathrm{ml}$ bottles. Once the centrifugation was completed, the centrates were added to cold ethanol in 1:2 ratio (supernatant:ethanol). These samples were kept refrigerated at least overnight for the polymers to precipitate in alcohol. The precipitates were recovered by centrifuging at $10000 \mathrm{G}, 4^{\circ} \mathrm{C}$ for 30 $\mathrm{min}$. The centrates were discarded and the polymer pellets were taken into evaporating crucibles and dried at $36 \pm 1^{\circ} \mathrm{C}$ after which their quantities were determined. Sludge pellets (at the bottom of the centrifuge bottles following the initial centrifuging) were resuspended in their own supernatant and taken into rheological tests. The described centrifugation run was conducted twice. At the end of each run sludge viscosity and quantity of extracted polymers were determined.

\section{Results and discussions}

\section{Rheological behaviour of activated sludge}

To investigate the rheological behaviour of activated sludge, shear stress vs. shear rate relationships were examined. At different solid concentrations the shear stress vs. shear rate graphs were plotted. One sample graph is presented in Fig. 1. This figure shows that the rheogram for activated sludge indicates non-Newtonian flow behaviour. When different rheological models (Newtonian vs. non-Newtonian) were tested for their fit to data, the best fit is obtained to a power law model. The $\mathrm{R}^{2}$ for a linear fit with zero intercept (Newtonian model) was 0.77 , whereas the $\mathrm{R}^{2}$ for a linear fit with a positive intercept (Bingham plastic model) was 0.96. On the other hand the $\mathrm{R}^{2}$ for the fit to power law model was 0.99 indicating that the flow of activated sludge can best be expressed by the pseudoplastic model, which is given by:

$$
\tau=\mathrm{K} \gamma^{\mathrm{n}}
$$

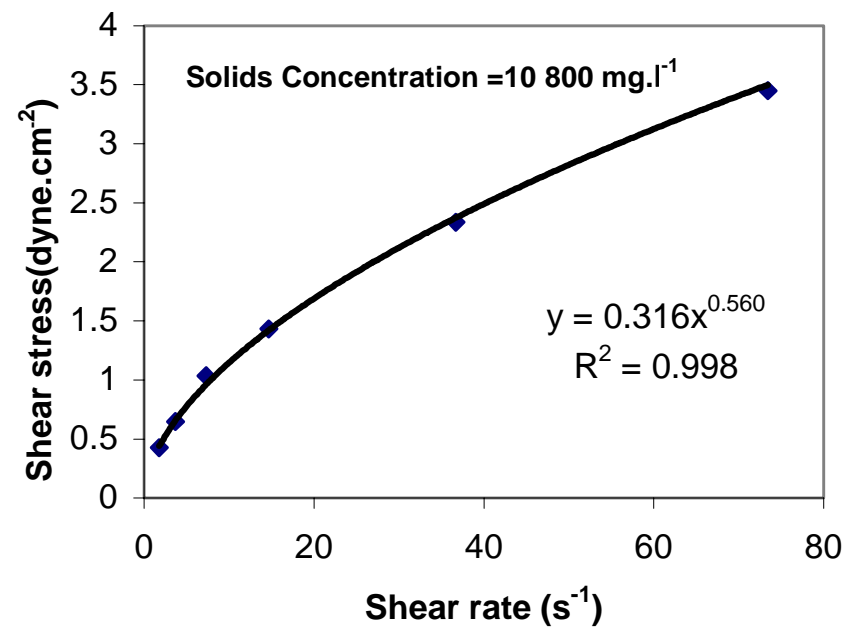

Figure 1

A sample rheogram for activated sludge

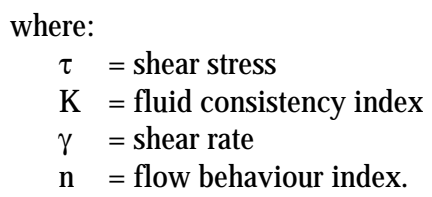

$\mathrm{K}$ is a term equivalent to viscosity and $\mathrm{n}$ is a number smaller than 1. Note that when $n=1$, the pseudoplastic model drops to Newtonian model. So as $\mathrm{n}$ gets smaller and smaller than 1 , the flow character deviates more and more from Newtonian.

Results of some earlier studies also indicate that the flow of activated sludge can best be expressed by the pseudoplastic model (Behn, 1962; Moeller and Torres, 1997).

\section{Effect of solids concentration of activated sludge on the rheological behaviour}

One of the factors that contribute to non-Newtonian flow behaviour of a fluid is the solids existing in that fluid. The presence and the concentration of solids have been given as the primary reasons for the increased viscosity in Einstein's Law of Viscosity (Hiemenz and Rajagopalan, 1997). Einstein's theory correlates the dispersion viscosity with the volume fraction of solids suspended in that fluid for spherical, non-interacting, unsolvated and rigid particles as follows:

$$
\eta / \eta_{\mathrm{o}}=1+2.5 \phi
$$

where:

$\eta=$ viscosity of the dispersion

$\eta_{\mathrm{o}}=$ viscosity of the dispersion medium

$\phi=$ volume fraction occupied by the particles .

Activated sludge being a two-phase slurry exhibits varying degrees of non-Newtonian behaviour depending on the slurry conditions. Figure 2 gives the rheograms of the same activated sludge at two different solids concentrations. For both concentrations, activated sludge exhibits a pseudoplastic flow character. When the effect of solids concentration on $\mathrm{K}$ and $\mathrm{n}$ values is examined, it can be said that the $\mathrm{K}$ value increases with the increase in solids concentration as expected. For a solids concentration of $3600 \mathrm{mg} \cdot \mathrm{l}^{-1}$, the $\mathrm{K}$ value is found to be 0.040 dyne $\cdot \mathrm{cm}^{-2} \cdot \mathrm{s}^{\mathrm{n}}$, whereas it increases to 


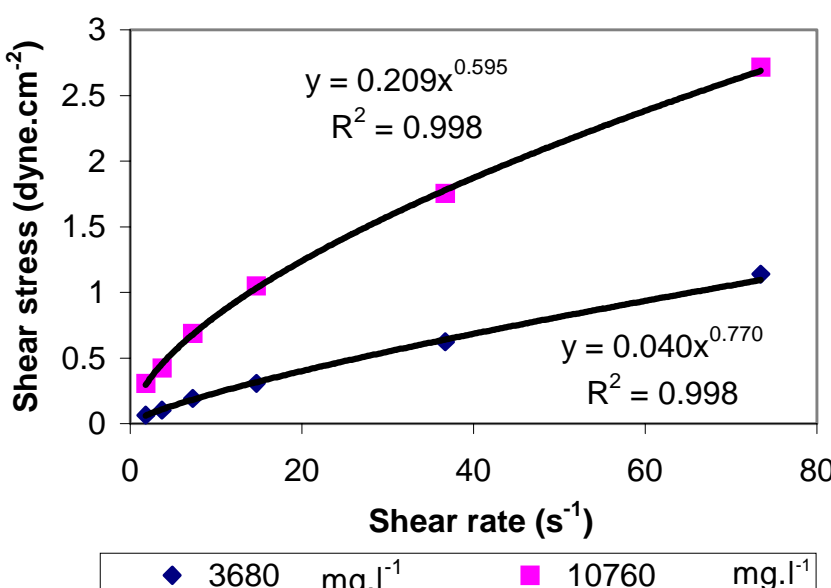

Figure 2

Rheograms of activated sludge at two different solids concentration

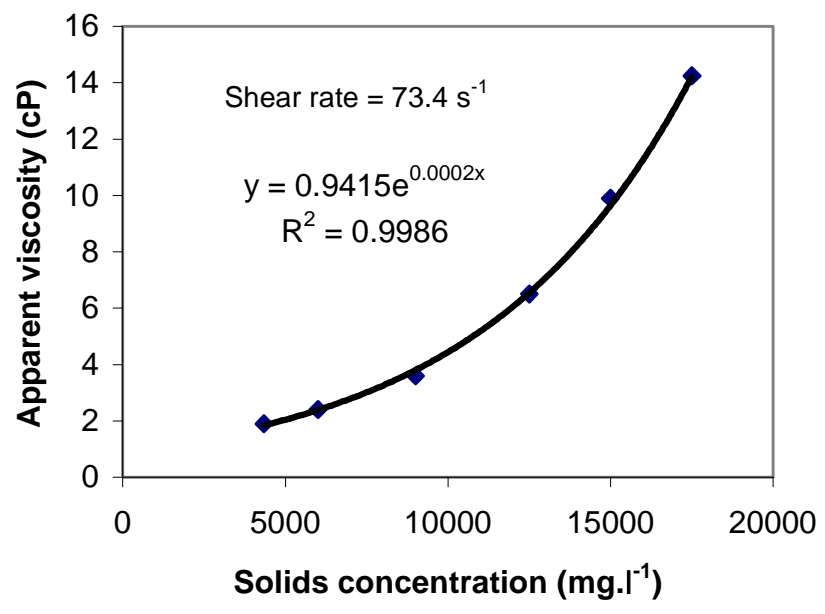

Figure 3

Effect of solids concentration on viscosity of activated sludge

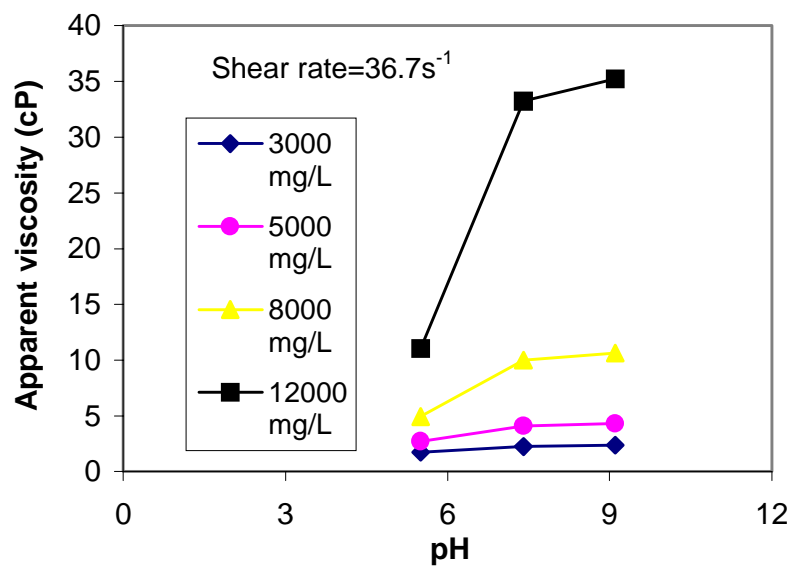

Figure 4

Effect of $\mathrm{pH}$ on viscosity of activated sludge
0.209 dyne $\cdot \mathrm{cm}^{-2} \cdot \mathrm{s}^{\mathrm{n}}$ when the solids concentration is increased to 10 $760 \mathrm{mg} \cdot \mathrm{I}^{-1}$. This simply indicates that the viscosity of the sludge increases significantly with increasing solids concentration. When the $n$ values of sludge at different solids concentrations are examined, a greater deviation from 1 is seen as the solids concentration of the sludge is increased. The $\mathrm{n}$ values are 0.770 and 0.595 at solids concentrations of $\left.3600 \mathrm{mg} \cdot\right|^{-1}$ and $\left.10760 \mathrm{mg} \cdot\right|^{-1}$, respectively. The decrease in $n$ value is another indicator that the flow of sludge deviates stronger from Newtonian behaviour as the solids concentration increases. Similar observations of $\mathrm{K}$ and $\mathrm{n}$ are reported by Lotito et al. (1997).

The variation of sludge viscosity with solids concentration is given in Fig. 3. The increase of apparent viscosity with solids concentration follows an exponential pattern. This explains the stronger non-Newtonian behaviour with increasing solids concentration. Some earlier researchers report the solids concentration and viscosity relationship in slightly different forms. For example one study reports that the $\mathrm{K}$ value (pseudoplastic viscosity) and the total solids concentration of activated sludge are related by a power law expression (Lotito et. al., 1997).

Obviously all the viscosity equations developed as a function of solids concentration in sludge research are quite different from the Einstein's Equation of Viscosity. However, it is also discussed that the deviations from Einstein's theory originate mainly in the systems where the concentration is high (volume fractions higher than 0.1) and the particles interact with each other (Hiemenz and Rajagopalan, 1997). Considering that sludge is a microbial slurry where the particles constantly interact with each other, it would be unrealistic to expect that Einstein's theory would be obeyed.

\section{Effect of $\mathrm{pH}$ on the rheological properties of activated sludge}

To analyse the effect of $\mathrm{pH}$, viscosities measured for sludge at different $\mathrm{pH}$ values at various solids concentrations are plotted in Fig. 4. This figure shows that at every solids concentration, the lowest viscosity is attained when the $\mathrm{pH}$ of the system is at 5.5. As the $\mathrm{pH}$ is increased to 7.4, the viscosity measured increases. This effect is intensified as the solids concentration is increased. Activated sludge at $\mathrm{pH} 9.1$ does not behave much different from activated sludge at $\mathrm{pH} 7.4$ for most solids concentrations examined.

Considering that the isoelectric point of bacteria is at $\mathrm{pH} 2$ to 4 (Tenney and Stumm, 1965) it is clear that sludge particle surfaces would carry increasingly negative charges as the $\mathrm{pH}$ is increased above the isoelectric point; $\mathrm{pH} 5.5$ is the value closest to the isoelectric point of bacteria. Therefore, at this $\mathrm{pH}$, the floc surface is only slightly negatively charged. With increasing $\mathrm{pH}$ values the floc system will be increasingly negatively charged. Increasing similar charges in the floc structure causes a repulsion and an expanded floc matrix at high $\mathrm{pH}$ values. On the other hand, at $\mathrm{pH}$ 5.5 , the floc structure is at its most compact form. Whether the flocs are compact or expanded makes a difference in the fluid flow and deformation properties. An expanded floc structure (at $\mathrm{pH} 7.4$ or 9.1 for example) creates a higher resistance to flow due to an increase in the exposed cross-sectional area. This in turn creates a higher viscosity in the system. On the other hand, when the flocs are more compact like at $\mathrm{pH} 5.5$, the exposed cross-sectional area of solids is lower, therefore the obstruction of flow lines will be less. This reflects as a lower viscosity. As can be seen in Fig. 4, the $\mathrm{pH}$ effect is intensified by the increasing solids concentration. This is expected since the viscosity relationship with solids concentration is already shown to be an exponential relationship. 


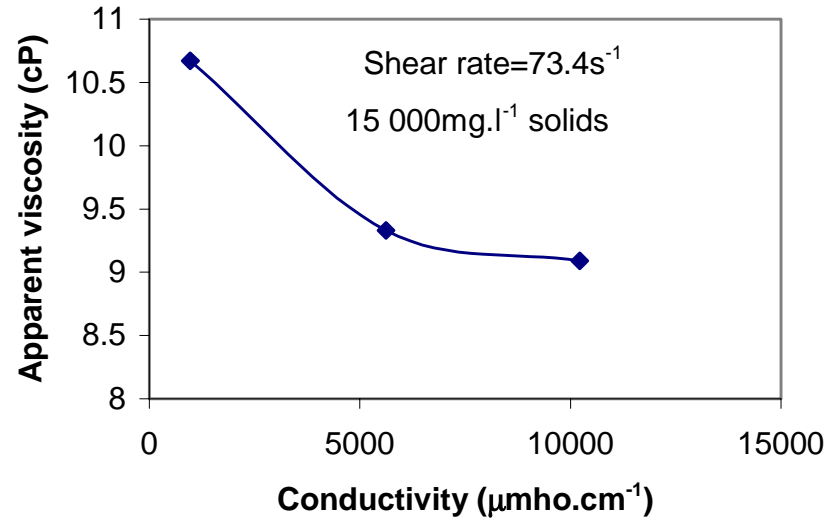

Figure 5

Effect of conductivity on viscosity of activated sludge

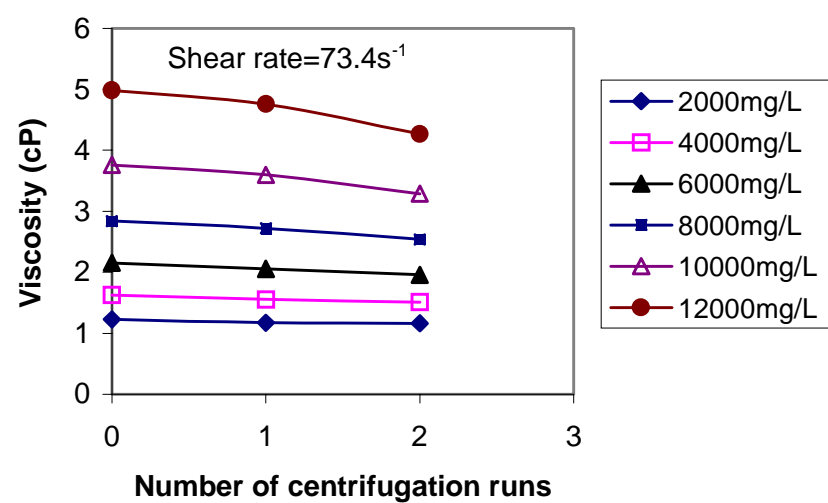

Figure 6

Effect of centrifugation at $16500 \mathrm{G}$ on sludge viscosity

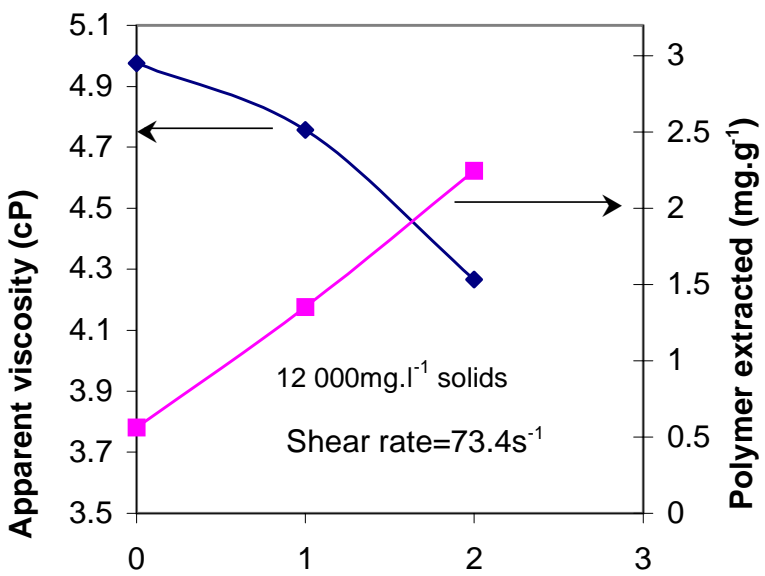

Number of centrifugation runs

Figure 7

Relationship between the number of centrifugation runs viscosity and the amount of polymer extracted from sludge

\section{Effect of ion concentration on the rheological properties of sludge}

Another important chemical factor that may bring out a similar effect to $\mathrm{pH}$ on viscosity is the ionic strength. Ionic strength affects the thickness of the electrical double layer around the charged interfaces as well as modifying the spatial extension, i.e. the conformation, of polymers attached to the surfaces. The experiments in this part have been conducted by adjusting the conductivity of activated sludge at a fixed solids content $\left(\left.15000 \mathrm{mg} \cdot\right|^{-1}\right)$ with the addition of various amounts of ions of a buffer solution. The original conductivity of an activated sludge sample is 970 $\mu \mathrm{mho} \cdot \mathrm{cm}^{-1}$. When this activated sludge is added the salts constituting the typical phosphate buffer saline (PBS) described previously, the measured conductivity becomes $5620 \mu \mathrm{mho} \cdot \mathrm{cm}^{-1}$. In the third case, twice the amount of PBS salt solution is added to the same activated sludge and this sample has a conductivity of 10220 $\mu \mathrm{mho} \cdot \mathrm{cm}^{-1}$. The viscosities of these 3 samples have been measured and are presented in Fig. 5, which shows that with the increase in conductivity, there is a decrease in viscosity. This decrease is sharper especially in the earlier part of the graph when the conductivity is first increased. Then as more and more ions are added to the system, viscosity starts to give less response, changing minimally. As the electrical double layer is compressed at high ionic strengths, particles and flocs may assume closer localities with each other. In addition, the surface polymers of sludge (extracellular polymers) will assume a coiled conformation rather than a stretched conformation when the ionic strength of the medium is high. This makes the flocs more compact. In addition, smaller amounts of polymers will be extending out into the solution since their charges are screened out. On the contrary, at low ionic strengths, the floc structure is expanded, the negatively charged polymers are stretched far out into the solution since their charges are not screened out. Obviously the second case with the low ionic strength is expected to create a higher viscosity since this type of a system creates a higher resistance during flow as discussed earlier. This is exactly what has been observed in this study. A similar result is also reported earlier (Forster, 1982); with the addition of $\mathrm{NaCl}$ to activated sludge samples, the viscosity of the system is lowered. Futhermore, when high salt concentrations are added, the bound water content of the sludge decreases. The bound water content and the viscosity of the sludge have previously been positively correlated (Forster, 1983). Therefore, the mechanism of the effect of increasing ionic strength on the viscosity may also be through a decrease in the amount of bound water content which further causes a decrease in viscosity.

\section{Effect of extracellular polymers and flocculation on the rheology of activated sludge}

Considering that polymers are high-molecular mass and highviscosity substances and also that they act as glues in holding activated sludge particles together, the effects of polymers on sludge viscosity are investigated next. Earlier work revealed that extracellular polymers affect the viscosity of sludge (Forster, 1982; Forster, 1983) and also centrifugation especially at low speeds (2 $000 \mathrm{G}$ compared to $16500 \mathrm{G}$ for example) can extract some extracellular polymers from activated sludge (Sanin and Vesilind, 1994). Multiple centrifugation runs are employed in this work to examine the effect of centrifugation on viscosity and observe if there is any correlation to the amount of polymers extracted. Centrifugation may affect the viscosity by two mechanisms: 
- Simply breaking the activated sludge floc structure; and

- First extracting the polymers and then breaking up the flocs.

These two mechanisms are inseparable in most cases: centrifugation breaks the flocs and some polymers are released into the medium. Figure 6 shows that with centrifugation the viscosity of the sludge can be lowered to a certain extent. This effect is seen both when the same sludge is centrifuged once and twice; and it becomes more pronounced after a solids concentration of 6000 $\mathrm{mg} \cdot \mathrm{l}^{-1}$. At the highest solids concentration investigated (12 000 $\mathrm{mg} \cdot \mathrm{I}^{-1}$ ) there is about $16 \%$ decrease in viscosity at the end of the second centrifugation run at $2000 \mathrm{G}$.

Figure 7 shows once again that the decrease in the viscosity of sludge correlates well with the amount of polymers extracted, indicating that there is a relationship between the polymer quantity and viscosity. This shows that the flocculated nature of activated sludge is critical in determining the rheological properties, which has significant implications in sludge pumping and pipeline transport.

\section{Conclusions}

In line with some earlier findings, activated sludge is found to behave as a pseudoplastic fluid in this study. From among the parameters studied, solids concentration has the most significant effect on the rheological property of sludge. With increasing solids concentration, pseudoplasic viscosity increases sharply and the flow behaviour index decreases significantly indicating that the flow tends to be more pronounced non-Newtonian at high solids contents. This is an important concern in the design of transport systems for thick sludges. Solution $\mathrm{pH}$ affects the apparent viscosity of sludge. Lowest viscosity is observed at the lowest $\mathrm{pH}$ studied (5.5) which is a value closest to the isoelectric point of bacteria. With increasing $\mathrm{pH}$ values, the viscosities are observed to increase. Effect of $\mathrm{pH}$ is significantly intensified when the solids concentration is increased. Apparent viscosity is found to decrease with increasing conductivity of sludge. This is believed to be due to the compression of the electrical double layer around the particles that results in a more compact floc structure and coiled polymer confomation in and out of the flocs. Presence of extracellular polymers affects the viscosity of sludge. Removal of polymers by multiple centrifugation runs correlates well with the decrease in viscosity. Even though all the parameters studied showed significant impact on activated sludge viscosity, the primary parameter determining the viscosity as well as the degree of nonNewtonian behaviour is the concentration of solids.

\section{References}

BEHN VC (1962) Experimental determination of sludge flow parameters. J. Sanitary Eng. Div., Proc. ASCE 88 (SA3) 39-54.

DICK RI and BUCK JH (1985) Measurement of activated sludge rheology. In: ASCE Env. Eng. Proc. of the 1985 Specialty Conference, Boston, MA, July 1-5.

DICK RI and EWING BB (1967) The rheology of activated sludge. JWPCF 39 (4) 543-560.

DICK RI (1986) Rheology of Biological Wastewater Suspensions. Report prepared for National Science Foundation, NSF/ENG-86044, Washington, DC, USA. 126 pp.

FORSTER CF (1983) Bound water in sewage sludges and its relationship to sludge surfaces and sludge viscosities. J. Chem. Technol. Biotechnol. 33B (1) 76-84.

FORSTER CF (1982) Sludge surfaces and their relation to the rheology of sewage sludge suspensions. J. Chem. Technol. Biotechnol. 32 (8) 799-807.

HIEMENZ PC and RAJAGOPALAN R(1997) Principles of Colloid and Surface Chemistry (3rd edn.) Marcel Dekker, Inc., New York. 650 pp.

LOTITO V, SPINOSA L, MININI G and ANTONACCI R (1997) The rheology of sewage sludge at different steps of treatment. Water Sci. Technol. 36 (11) 79-85.

MOELLER G and TORRES LG (1997) Rheological characterization of primary and secondary sludges treated by both aerobic and anaerobic digestion. Bioresource Technol. 61 (8) 207-211.

SANIN FD and VESILIND PA (1994) Effect of centrifugation on the removal of extracellular polymers and physical properties of activated sludge. Water Sci. Technol. 30 (8) 117-127.

SHAW DJ (1992) Introduction to Colloid and Surface Chemistry (4th edn.). Butterworth Heinemann, Oxford. 306 pp.

TENNEY MW and STUMM W (1965) Chemical flocculation of microorganisms in biological waste treatment. JWPCF 37 (10) 1370-1388.

UNNO $\mathrm{H}$ and AKEHATA $\mathrm{T}$ (1985) Some rheological features of concentrated excess activated sludge of thixotrophic nature. J. Chem. Eng. Japan 18 (6) 533-538. 
